\title{
Bilateral intraorbital hemorrhage with factor XIII deficiency after strabismus surgery
}

\author{
Erbil Seven ${ }^{1^{*}}$, Muhammed Batur $^{1}$, Adnan Çınal ${ }^{2},{\text { Tekin } \text { Yaşar }^{1}, \text { Ahmet Demirok }}^{3}$ \\ ${ }^{1}$ Department of Ophthalmology, Yuzuncu Yil University, Van, Turkey \\ 2Department of Ophthalmology, Gazi University, Ankara, Turkey \\ ${ }^{3}$ Department of Ophthalmology, Medeniyet University, Istanbul, Turkey
}

\begin{abstract}
Factor XIII deficiency is a very rare, inherited bleeding disorder. In this case, we report a child with intraorbital hemorrhage following strabismus surgery was diagnosed with factor XIII deficiency.
\end{abstract}

Key Words: Fibrin stabilizing factor, intraorbital hemorrhage, strabismus surgery

\section{Introduction}

Factor XIII deficiency (fibrin stabilizing factor) is an autosomal recessive inherited disorder with an estimated incidence of $1 / 2,000,000$ (1). No information is available in the current literature regarding factor XIII deficiency and ophthalmic surgery.

In this study, we report a child who underwent strabismus surgery and afterward developed serious bilateral intraorbital hemorrhage. The hemorrhage resulted to diagnosis of factor XIII deficiency.

To the best of our knowledge, this is the first reported case of intraorbital hemorrhage after strabismus surgery associated with factor XIII deficiency.

\section{Case report}

A 2-year-old girl had a 40 prism diopter infantile esotropia. She had no specific family history of ocular or systemic diseases. We planned surgery for correction of her strabismus. Routine laboratory tests performed before surgery, including prothrombin time, activated partial thromboplastin time, bleeding time, and a complete blood count, revealed no abnormality. Surgery involved an uneventful $5.5 \mathrm{~mm}$ bilateral medial rectus recession using two single-armed 6.0 vicryl sutures through limbal conjunctival incisions under general anesthesia. The visible vessels were cauterized for hemostasis during the operation. No noticeable findings were evident during the operation. The strabismus surgery and immediate postoperative convalescence were uncomplicated.

Four hours after surgery, the onset of swelling and periorbital discomfort was noted on the right side, with a fresh hemorrhage from the right medial conjunctival incision. Twenty-four hours after surgery, an intraorbital hemorrhage, a massive chemosis, tense eyelids, complete ptosis, and an exotropia were seen in her right eye. In the left eye, the clinical picture was less dramatic, but a fresh hemorrhage from the left medial conjunctival incision, chemosis, ptosis, and mild exoptropia were noted. Orbital computed tomography (CT) scanning was immediately performed and showed a nasal intraorbital hematoma, which continued along the medial rectus muscle and compressed the globe at the antero-medial region in right eye. The bleeding in the left eye showed no noticeable findings on CT at that time.

The hematological laboratory tests were repeated twice but were still normal. Nevertheless, we suspected some form of coagulopathy. Therefore, the patient underwent a pediatric hematology consultation.

Parenteral fresh frozen plasma infusion was started to stop the eye bleeding and orbital compression. Following fresh plasma infusion, the eye bleeding stopped immediately. The clinical findings were markedly resolved by the $5^{\text {th }}$ day of the treatment (Figure 1) and were almost healed at the $13^{\text {th }}$ day. 


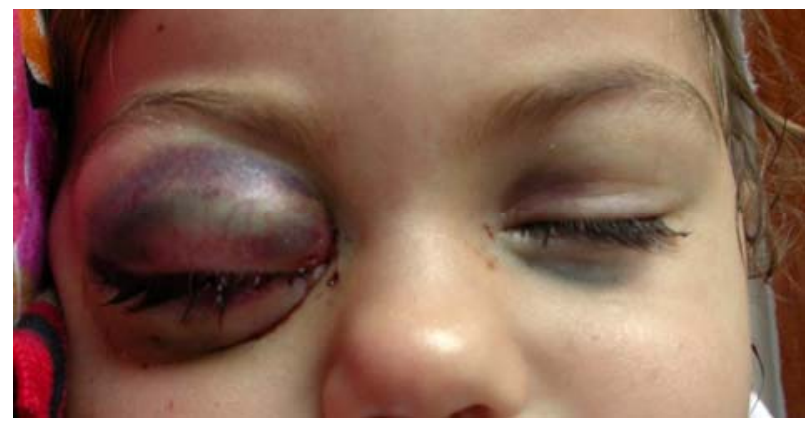

Fig. 1. The patient on postoperative day 5, showing proptosis, lid swelling and chemosis.

We considered a clotting disorder and hematologic studies revealed a factor XIII deficiency. Other clotting factors and coagulation tests were normal. Factor XIII deficiency was diagnosed by the clot solubility assay with acetic acid. We learned that the patient had a history of easy, prolonged, and repeated bruising. There was no history of miscarriage, central nervous system stroke, or hemorrhage in the family.

The patient had normal ophthalmological features at postoperative 6 months (Figure 2).

Two years later, the patient's newborn sister also was diagnosed with factor XIII deficiency because of the family awareness.

\section{Discussion}

Intraorbital hemorrhages are rare in the pediatric population. They have a wide range of etiologies, including trauma and surgery (2-4), but factor XIII deficiency is not a reported cause.

We report this disease as a new etiologic factor for intraorbital hemorrhage. Activated partial thromboplastin time and prothrombin time are routinely tested as part of the surgery preparation of patients with no noticeable history of disease in our clinics. Because these tests are normal in patients with factor XIII deficiency (1), we did not diagnose factor XIII deficiency preoperatively in our patient.

Bleeding at the time of surgery may be minimal in factor XIII deficiency, but delayed hemorrhage often occurs because of unstable fibrin clots (1). The bleeding in our case started 4 hours after surgery, which can be interpreted as delayed hemorrhage.

Postoperative bleeding from the eye may occur due to insufficient perioperative hemostasis and cauterization. However, in the current case, the bleeding was too abundant to be explained solely

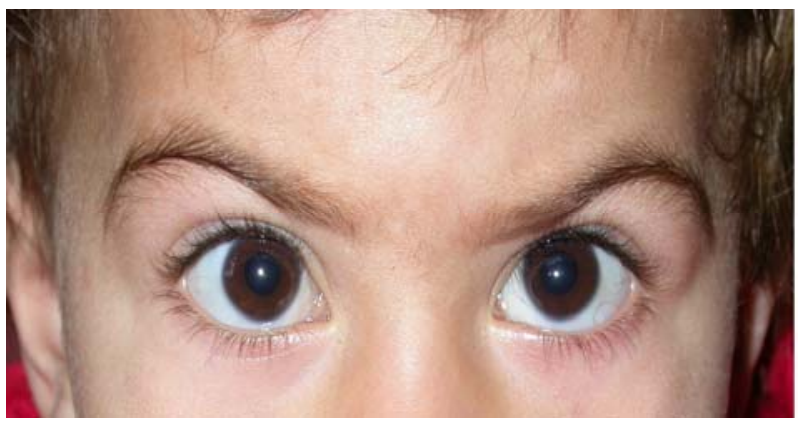

Fig. 2. The patient on postoperative 6 months with normal clinical features.

by insufficient hemostasis. Therefore, we considered other explanations for the bleeding and requested $\mathrm{CT}$ and pediatric consultations. CT scans showed that the bleeding arose from the medial rectus muscle, and pediatric hematology confirmed the diagnosis as factor XIII deficiency. We treated the bleeding with a fresh frozen plasma infusion.

Prompt treatment of these cases might prevent further damage, such as corneal exposure damage and compressive optic neuropathy. The wrong management of these cases would be reoperation for hemostasis with the view that the bleeding arises from insufficient cauterization, as this could cause further bleeding and more serious complications.

Factor XIII deficiency presenting following strabismus surgery, with no remarkable patient and family history, must be very rare. To the best of our knowledge, no bilateral orbital fresh bleeding with factor XIII deficiency has been reported after strabismus surgery in English language papers in the literature.

Ophthalmologists should be aware that intraorbital hemorrhage may develop after surgery in patients with routine preoperative hematologic results associated with preoperatively undiagnosed factor XIII deficiency.

Conflict of interest: The authors have no financial or personal relationships with people or organizations that could potentially and inappropriately influence their work.

\section{References}

1. Gailani D and Neff AT. Rare Coagulation Factor Deficiencies. In: Hematology: Basic Principles and Practice, Eds: Hoffman R, Benz EJ, Silberstein LE, Heslop HE, Weitz JI, Anastazi J, Sixth Edition, Chapter 139, Elsevier Inc, 2013. 
2. Costa Jr LB, Andrade A, Henriques JGB, Cordeiro AF, Maciel CJJ. Traumatic bilateral intraorbital (subperiosteal) hematoma associated with epidural hematoma Case report. Arq Neuropsiquiatr 2003; 61: 1039-1041.
3. Todd B, Sullivan TJ, Gole GA. Delayed orbital hemorrhage after routine strabismus surgery. Am J Ophthalmol 2001; 131: 818-819.

4. Morax S. Complications of blepharoplasty. J Fr Ophtalmol 2004; 27: 658-674. 